\title{
On the contribution of charge-exchange induced X-ray emission in the ISM and ICM
}

\author{
R. Lallement
}

\author{
Service d'Aéronomie du CNRS, 91371 Verrières-le-Buisson, France \\ e-mail: rosine.lallement@aerov.jussieu.fr
}

Received 4 November 2003 / Accepted 11 May 2004

\begin{abstract}
X-ray emission can be generated by charge-exchange (CXE) between highly charged ions of a hot plasma and neutral species of an interacting cool/warm gas, a phenomenon recently observed in the case of the solar wind interaction with cometary, interstellar, and geocoronal neutrals (Lisse et al. 1996; Cox 1998; Cravens 1997, 2002).

Charge-exchange processes are included in most theoretical models of hot interstellar plasmas interacting with partially neutral gas, resulting in a modification of the physical states of the gases in the interaction region. However, the contribution of the charge-exchange induced X-ray emission produced at these interfaces has hitherto not been considered to be significant. The detailed calculations performed by Wise \& Sarazin (1989), motivated by the observations of X-ray emission following chargeexchange in laboratory fusion devices, have shown that the emission is negligible in the case of an SNR fast shock. Our goal here is to investigate its relative importance in different astrophysical cases. We simplistically consider interfaces between partially neutral and hot gas in the following cases: (a) a supernova blast wave propagating in a neutral (or partially ionized) ISM (b) a galactic wind engulfing a halo dense cloud; (c) a high velocity cloud (HVC) moving through the halo, and (c) a dense cloud moving in intra-cluster gas. Although the phenomenon is restricted to the very narrow envelopes defined by the mean free path of the neutrals through the hot plasma, it is easy to show that its efficiency is such that the volume emissivity from these interfaces can be orders of magnitude above the emissivity of the hot gas itself, and, more important, that its relative contribution increases with decreasing hot gas density. As a consequence it should be at maximum in mixing layers in very low density hot gas.

Our preliminary results suggest that the charge-exchange X-ray emission from the interface does not contribute significantly in case (a), in agreement with Wise \& Sarazin, except marginally for lines of sight tangent to the interfaces, but that it can be a non-negligible fraction of the hot gas emission in cases (b-d). Detailed self-consistent models of plasma and neutral atom distributions in conduction fronts or contact discontinuities are needed for better estimates.

In the easiest test case of $\mathrm{HVCs}$, there is a good agreement between our predicted equivalent emission measure $E M \approx$ $0.01 \mathrm{~cm}^{-6} \mathrm{pc}$ for complex C-type clouds and their soft X-ray emission measured by ROSAT (e.g. Kerp et al. 1996).

If confirmed by more realistic calculations, a contribution of the CXE emission may play a role in a number of astrophysical cases: (i) tight correlations between $\mathrm{H} \alpha$ and X-ray patterns; (ii) enhanced X-ray limb brightening at interfaces; (iii) spectral and abundance "anomalies", because charge-exchange spectra differ from thermal spectra and contain only emission lines, resulting in some biases when they are interpreted with classical models.

Interestingly, interaction between cold IS clouds and the intra-cluster gas finally represents the most favorable case for the CXE emission, with the following potential consequences. At low spectral resolution, CXE emission can mimic global hot gas cooling. However, it is not in fact a global cooling, but instead accelerated cooling restricted to small physical areas, associated with the relative motions of the neutral clouds.

In galaxy clusters, if CXE emission is a non-negligible contributor to the total X-ray diffuse emission, it may help reduce the need for cooling flows.
\end{abstract}

Key words. ISM: clouds - ISM: general - Galaxy: halo - galaxies: starburst - galaxies: cooling flows stars: supernovae: general

\section{Introduction}

50 years after the discovery of the solar wind from cometary tails, comets have yet again revealed an unexpectedly important physical phenomenon, i.e. EUV and X-ray emission from neutral-solar wind interaction (Lisse et al. 1996).
Charge-exchange X-ray emission (CXE) is currently observed in laboratory fusion devices utilizing strong neutral beams, but previously its role in astrophysics had not been considered to be significant, mainly because astrophysical objects emitting X-rays are associated with large quantities of very hot plasma, and the thermal emission of the hot gas is supposedly greater than the emission from the interfaces with the cooler 
media. Indeed, in a pioneering work, Wise \& Sarazin (1989) estimated the CXE emission from the periphery of a SNR fast shock, and found it to be negligible (but by only one or two orders of magnitude in some energy ranges) compared to the emission from the shock-heated gas within the remnants.

In the solar wind-comet interaction, the CXE mechanism is now well established, and found to be caused by charge transfer between solar wind high ions and neutrals from outgassed cometary material (Cravens 1997). The complex series of de-excitation cascades following electron-ion recombination produces a spectrum which is characteristic of the solar wind ionic composition, i.e. abundances and ionization states (e.g. Kharchenko \& Dalgarno 2000; Schwadron \& Cravens 2002). The X-ray diffuse emission arises in the cometary cloud close to the nucleus, where solar wind ions encounter neutral atoms or molecules.

In parallel, the excellent correlation between the ROSAT non-cosmic enhancements of X-ray emission and the solar wind flux has revealed the heliospheric nature of this timevariable emission (Freyberg 1994). Thus, not only comets but other neutral species present in the heliosphere can generate X-rays after interaction with solar wind superions (Cox 1998). It has been shown by Cravens et al. (2001) and Robertson et al. (2001) that both the geocorona and the interstellar gas within the solar system are responsible for the ROSAT Long Term X-ray Enhancements, the so-called LTE's. Using numbers derived from cometary emissions, Cravens et al. (2000) have also suggested that a non-negligible fraction of the ROSAT R1/R2 diffuse emission could be of heliospheric origin. As a matter of fact, the sun is embedded in interstellar neutral gas and the emission associated with solar wind ions and neutrals must originate from the entire sky. The comparison between a model map of the emission appropriate to the ROSAT survey geometry and chronology on one hand, and the Local Bubble (LB) soft X-ray diffuse emission of Snowden et al. (1998) on the other hand, favors a substantial heliospheric contamination (Lallement 2003). As a positive consequence, subtracting a local solar background from the soft X-ray signal has the result of it attenuating the LB hot gas emissivity, and removes partially the long-standing discrepancy between the pressure of the LB hot gas directly derived from the soft X-ray emission (Snowden et al. 1995, 1998) and the measured pressure in the diffuse cloudlets embedded in the LB, in particular the Local Cloud through which the Sun is moving (Jenkins 2002; Lallement 1996).

It is remarkable that the level of the diffuse heliospheric $\mathrm{X}$-ray emission, whose major part is generated over a distance as short as $\simeq 10 \mathrm{AU}$ (where the interstellar gas interacts with a strong enough solar wind flux), is of the same order as the diffuse emission generated by the Local Bubble hot gas over hundreds of parsecs. This is due to the efficiency of the chargeexchange process, the high solar wind ion flux in the inner heliosphere, and our location within the emissive region, which implies that all lines of sight (LOS) cross the entire interaction region.

Schwadron \& Cravens (2000), and Kharchenko \& Dalgarno (2000) have estimated the spectral characteristics of the CXE emission in the soft X-ray domain. Emission lines are dependent on the dominant charge states and abundances in the solar wind, i.e. on the solar wind type. Some of the lines are stronger and others fainter than in the recombination spectra derived from the classical models.

Recently, with the Chandra and XMM satellites in operation, a number of highly spatially and spectrally resolved observations in X-rays have revealed new features, correlations and spectral properties of hot gas emission from SNR's, galactic halos and galaxy clusters. In particular, the spatial correlation between $\mathrm{H}-\alpha$ and X-ray enhancements appears to be a general feature in many types of astronomical objects. Such X-ray enhancements are in general explained in terms of shocked and heated gas associated with expansion of SNR waves, galactic winds or ICM hot gas into cool clouds. However, the new observations are sometimes difficult to interpret. In a number of studies of star forming galaxies, the spectra of the halos are characterized by a multi-temperature structure, including an unexplained "soft" component (e.g. Strickland et al. 2002). On the other hand, they are characterized by unrealistically low metal abundances, when interpreted in the frame of monoor multi- temperature thermal models. CXE spectra contribute only lines, and thus in principle cannot explain low abundances, unless the CXE different relative line strengths play a strong and complicated role in the interpretation.

In the case of galaxy clusters, the new high quality spectra are in clear disagreement with the cooling flow models (e.g. Peterson et al. 2003). The possibility of a contribution to the total diffuse emission by charge-exchange induced emission generated in neutral gas-hot plasma interfaces is investigated here. Because self-consistent models of both plasma and neutrals at traveling shocks or contact discontinuities are in general not necessary for understanding the main physical properties of the fast shocks or expanding fronts, the development of such models has been restricted to a limited number of cases, for example as in the outer boundary of the heliosphere (Baranov \& Malama 1993; Izmodenov et al. 1999). These self-consistent models are complex because they associate hydrodynamic and kinetic descriptions (for the plasma and the atoms respectively). Results based on such plasma/neutrals models are thus far beyond the scope of this paper, whose limited goal is, at variance with the work of Wise \& Sarazin (1989), a preliminary and very crude estimate of the CXE emission, but not limited to the SNR blast wave case. We first derive, by analogy with the heliospheric emission, a formula for the maximal relative contribution of such an interface (Sect. 2). We then consider four different astrophysical configurations, illustrated by a well known astronomical object (except for case (d)), and for each case we use a simplistic model to estimate the possible contribution of the charge-exchange induced X-ray emission (Sects. 3-5). We find that in all four cases there may be a contribution to the total $\mathrm{X}$-ray emission, especially along lines of sight which are tangent to the surfaces forming the interface between hot expanding winds and cooler gas, but this contribution is significant only in the very low density gas of galactic halos and intracluster space. We suggest that this additional emission from the charge-exchange reactions could help explain some of the observed correlations, spectral properties and abundance anomalies presently observed in the X-ray spectra of some objects, 

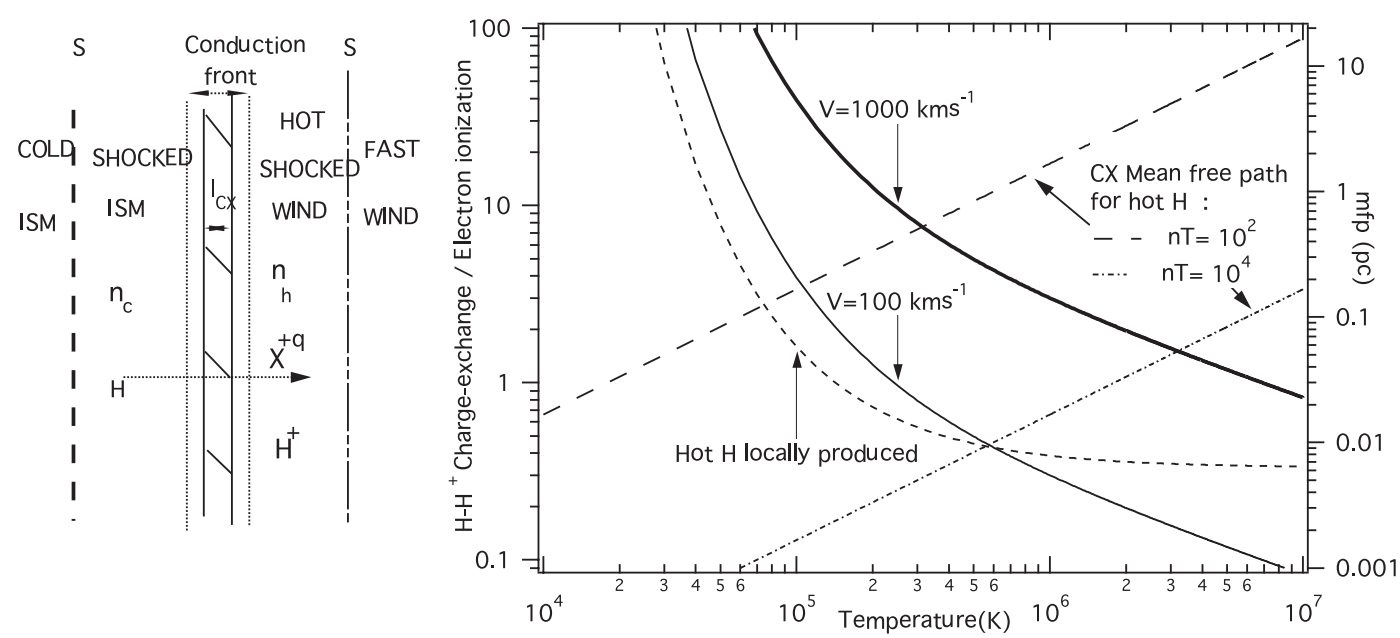

Fig. 1. Left: a general cold cloud/hot gas interface. Charge-exchange may occur in a narrow layer within the conductive front separating the cold and the hot gases. Right: ratio between charge exchange probability and electron collisional ionisation probability for an $\mathrm{H}$ atom streaming at $100 \mathrm{~km} \mathrm{~s}^{-1}$ (solid thin) and $1000 \mathrm{~km} \mathrm{~s}^{-1}$ (solid thick). Below $\approx 10^{5} \mathrm{~K}, \mathrm{CXE}$ is dominant and creates energetic neutrals. Above this temperature, $\mathrm{H}$ is preferentially ionized, but the ratio remains above 0.1 , suggesting that a non negligible fraction of the $\mathrm{H}$ atoms can survive and chargeexchange before ionization. The ratio is also shown for hot neutrals created locally by charge-exchange (dashed). Their mean free path (right scale) is shown for two different pressures.

and discuss the need for future investigations in this direction (Sect. 6).

\section{Soft X-ray emissivity due to charge-exchange processes at interfaces between partially neutral IS clouds and hot gas: Comparisons with hot gas emissivity}

We consider a general interface region between partially neutral gas (neutral density $n_{\mathrm{c}}$ ) and hot plasma $\left(n_{\mathrm{h}}, T_{\mathrm{h}}\right)$ impacting on the neutral gas (or reciprocally). This hot gas may be the shocked gas downstream of a shock wave, or the hot side of a conductive front separating a hot wind and a neutral cloud, or of a standing shock. A primary condition for CXE emission to occur is that the impacted gas is not completely photoionized by the astronomical object which produces the hot expanding gas, nor by its associated cosmic rays. This means either a large distance from the photoionizing sources, or weak sources. Ionization by a shock precursor has to be relatively weak too. This condition is fullfilled in a number of astrophysical cases, in particular when $\mathrm{H} \alpha$ emission is observed to be closely associated with hot gas X-ray emission. The second condition is that at such interfaces some of the neutrals may diffuse in the hot gas deeply enough to encounter high ions. The diffusion length scale is determined by the fastest plasmaneutral process, i.e. charge-exchange with protons or ions, and ionization by electron impact. Because charge transfer between neutral species and protons produce newly formed $\mathrm{H}$ atoms, it is not an ionization process and the new atoms are potentially still available for CXE. Not all energetic $\mathrm{H}$ atoms resulting from CXE, however, can participate in further chargeexchange, since these new atoms are linked dynamically to the hot plasma and will suffer collisional ionization. The relative importance of charge-exchange and electron impact ionization (we neglect collisional ionization by ions) depends on the temperature and on the relative velocity of the neutral flow. Figure 1 shows the probability ratio between the two processes as a function of temperature and the associated mean free paths in constant pressure conduction fronts. For velocities between 100 and $1000 \mathrm{~km} \mathrm{~s}^{-1}$ the ratio varies between 0.1 and 2 in the temperature range $10^{5}, 10^{7} \mathrm{~K}$. This implies that in sharp conduction fronts a non negligible fraction of the neutrals may charge-exchange with ions before getting collisionally ionized.

Since our goal is only to estimate the importance of CXE emission, in the following discussion we do not model the neutral and plasma density profiles across the interface, which depend on the type of hot/cool gas interaction. We assume that ionic stages and ion relative abundances are the same everywhere in the hot gas in the interaction region, which allows us to link the sum of all $\mathrm{CX}$ processes to the $\mathrm{H}-\mathrm{H}^{+}$chargeexchange process itself by a simple constant of proportionality. This assumption is sometimes questionable, in particular in the fast shock case, and will be discussed in the next sections. The thickness of the charge-exchange layer is estimated as the distance neutral $\mathrm{H}$ atoms can cover in the hot plasma, i.e. the mean free path for charge-exchange with $\mathrm{H}^{+}$if collisional ionisation is smaller, or the mean free path ( $\mathrm{mfp}$ ) for electron ionization in other cases. For simplicity in the formulation we define the factor $\epsilon$ as the ratio between the charge-exchange probability and the collisional ionization probability, i.e.

$\epsilon=\frac{\sigma n_{\mathrm{H}^{+}} V_{\mathrm{r}}}{q_{i} n_{\mathrm{e}}} \approx \frac{\sigma V_{\mathrm{r}}}{q_{i}}$

we keep the definition of the thickness of the layer as the mfp associated with the CX, and we replace the mean free path decrease by the factor $\epsilon$ by a decrease by the same factor of the density or neutrals able to fill the layer. $\epsilon$ is the fraction of the neutrals from the cool phase which can enter the shock or the contact discontinuity (CD) and charge-exchange before getting collisionally ionized. We also assume that the density 
of neutrals in this narrow layer is constant and equal to the average value $\epsilon n_{\mathrm{cx}}$ over the thickness $l_{\mathrm{cx}}$, and vanishes beyond.

In the case of an SNR fast shock (Sect. 3) almost all neutrals are reaching the post-shock gas and fill the narrow layer of width $l_{\mathrm{cx}}$, since $\epsilon \geq 1$, but in conduction fronts $\epsilon \leq 1$. In this latter case, the presence of a transverse magnetic field and its associated sharper temperature gradient should favor the diffusion of the neutrals through the gradient and increase $\epsilon$.

By defining the CX layer as the smallest of the mean free paths against charge-exchange with protons and collisional ionization respectively, the CX phenomenon may be underestimated, because energetic neutral $\mathrm{H}$ atoms created by the first charge-exchange reactions have large mean free paths and can diffuse more easily through the hot gas and reach very low density regions (see Fig. 1). This type of effect however can be estimated by means of kinetic models only. On the other hand, if there are intermediate layers between the two gases, $\epsilon$ is smaller.

We thus consider the streaming through the narrow layer of thickness $l_{\mathrm{cx}}$ and density $n_{\mathrm{cx}}$ of a hot gas flow of flux $\varphi_{\mathrm{w}}=n_{\mathrm{h}} V_{\mathrm{r}}$, where $V_{\mathrm{r}}$ is the relative velocity between the cool-partially neutral gas and the hot gas and $n_{\mathrm{h}}$ is the hot gas density. Our goal is to estimate the X-ray emission originating in the narrow layer of neutrals diffusing through the plasma, and to compare with the emission of the hot plasma itself, i.e. from the entire volume it occupies.

These configurations are very different from the heliospheric case. The heliosheath is stationary and open, the hot plasma is only slightly confined. More important, the expanding solar wind is so weak that interstellar neutrals from the local interstellar cloud cross the whole interface between the cloud and the wind, i.e. the shocked IS gas, the contact discontinuity and the shocked solar wind, and succeed in penetrating deep into the heliosphere, within a few AU. It is there that the solar wind flux becomes high enough for the charge-exchange to be able to fully ionize the neutral gas and produce X-rays. Because the solar wind is confined within a small volume, we have not considered the solar wind thermal emission. Instead, in the introduction we have compared the CXE emission with the emission originating in the hot gas filling the Local Bubble, and not with the solar plasma emission. If the solar wind had a flux many orders of magnitude above its actual value, and were expanding into a neutral ISM, its interface with this ISM, i.e., the CXE production area, would be at very large distance from the star. In this imaginary situation, comparing the surface brightness of the interface layer on the one hand, and the entire solar plasmasphere brightness on the other, is similar to what we intend to do in the next sections.

The volume emissivity $P_{\mathrm{cx}}$ due to charge-exchange processes is proportional to the hot gas flux through the neutrals (or reciprocally) and to $\Sigma_{x}$, the global emissivity cross-section for charge-exchange between neutrals ( $\mathrm{H}$ and $\mathrm{He}$ ) and all kinds of superions capable of producing X-rays contained in the hot gas. $\Sigma_{x}$ contains all the detailed physical processes during the cascades following the recombinations and the relative abundances of ions, and is the average X-ray emission per neutral $\mathrm{H}$ atom for a hot gas proton flux of unity. In the case of the solar wind $\Sigma_{x(\mathrm{SW})}=\Sigma_{0}$ is of the order of $6 \times 10^{-19} \mathrm{keV} \mathrm{cm}^{2}$ in the soft X-ray range (0.1-0.5 keV) (Schwadron \& Cravens 2000; Kharchenko \& Dalgarno 2000), which is the wavelength range we will consider throughout the following examples because of the availability of accurate intensity and spectral measurements of the CX process (estimates in another range, harder X-ray or on the contrary EUV can be obtained by changing the global cross-section). In the solar wind case $\Sigma_{0}$ does not depend on the distance to the sun because beyond a few solar radii high ions are "frozen" in the expanding gas. In other situations $\Sigma$ applies to the hot gas at the interface itself. As the next simplification, we assume that for a given hot expanding plasma $\Sigma_{x}=\Sigma_{0} \alpha$, where $\alpha$ accounts for the hot ion content, i.e. temperature and metallicity. For million degree gas with solar metallicity $\alpha$ is of the order of unity. We can then write the X-ray volume emissivity as:

$P_{\mathrm{cx}}=\Sigma_{0} \epsilon \alpha n_{\mathrm{c}} \varphi_{\mathrm{w}}=6 \times 10^{-19} \epsilon \alpha n_{\mathrm{c}} \varphi_{\mathrm{w}} \mathrm{keV} \mathrm{cm}^{-3} \mathrm{~s}^{-1}$

The order of magnitude of the emissivity can be illustrated by the following example: if $n_{\mathrm{c}}=1 \mathrm{~cm}^{-3}$ and $\varphi_{\mathrm{w}}=10^{6} \mathrm{~cm}^{-2} \mathrm{~s}^{-1}$ (as in the case of a galactic superwind at a few kpc impacting on dense gas), then $P_{\mathrm{cx}}=\alpha \epsilon 6 \times 10^{-11} \mathrm{keV} \mathrm{cm}^{-3} \mathrm{~s}^{-1}$. For comparison, the total (lines+continuum) volume emissivity of hot gas with a temperature $T=10^{6} \mathrm{~K}$ and density $n_{\mathrm{e}}=10^{-2} \mathrm{~cm}^{-3}$ is of the order of $10^{-18} \mathrm{keV} \mathrm{cm}^{-3} \mathrm{~s}^{-1}$ in the $0.1-0.5 \mathrm{keV}$ range. This shows that, depending on neutral densities in the interaction layer and and fluxes of plasma through the interface, the volume emissivity can potentially be orders of magnitude above the hot gas total emissivity.

In the following we estimate the balance between the two types of emission, CX emission and thermal hot gas emission in the simplified way:

$P_{\mathrm{cx}}=6 \times 10^{-19} \epsilon \alpha n_{\mathrm{c}} n_{\mathrm{e}} V_{\mathrm{r}} \mathrm{keV} \mathrm{cm} \mathrm{cm}^{-3} \mathrm{~s}^{-1}$

and

$P_{\mathrm{h}}=5.8 \times 10^{-14}\left(n_{\mathrm{e}}^{2}\right) \chi(T) \mathrm{keV} \mathrm{s}^{-1} \mathrm{~cm}^{-3}$

where $\chi(T, a)$ accounts for the ratio between the volume emissivity of hot gas at temperature $T$ and metal abundance $a$ ( $a$ in units of solar abundance) on one hand, and the emissivity of hot gas at 1 million $\mathrm{K}$ and solar abundance on the other hand. The volume emissivity ratio is:

$P_{\mathrm{cx}} / P_{\mathrm{h}}=10^{-5}\left(\epsilon \alpha \chi_{T, a}^{-1}\right) n_{\mathrm{c}} n_{\mathrm{e}}^{-1} V_{\mathrm{r}}$

The mean free path $l_{\mathrm{cx}}$ against charge-exchange for $\mathrm{H}$ is of the order $1 / \sigma n_{\mathrm{h}}$, where $\sigma$ is the charge-exchange cross-section for the H-proton reaction. For relative velocities of the order of $100-1000 \mathrm{~km} \mathrm{~s}^{-1}, \sigma \approx 1 \times 10^{-15} \mathrm{~cm}^{2}$. The CXE emission integrated along the distance $l_{\mathrm{cx}}$ can thus be estimated as:

$$
\begin{aligned}
P_{\mathrm{cx}} l_{\mathrm{cx}} & \approx 6 \times 10^{-19} \epsilon \alpha n_{\mathrm{c}} n_{\mathrm{e}} V_{\mathrm{r}} /\left(\sigma n_{\mathrm{e}}\right) \\
& \approx 0.6 \times 10^{-3} \epsilon \alpha n_{\mathrm{c}} V_{\mathrm{r}} \mathrm{keV} \mathrm{cm}^{-2} \mathrm{~s}^{-1}
\end{aligned}
$$

and, except for the factor $\epsilon$, is independent of the hot gas density, because by definition neutrals (the fraction which escapes collisional ionization) always charge-exchange with about the same column of protons (and their associated minor ions) when they cover a distance equal to the charge-exchange mfp. 
If $l_{\mathrm{h}}$ is the path length for thermal emission along the LOS, then the CXE emission becomes important if $P_{\mathrm{cx}} L_{\mathrm{cx}} / P_{\mathrm{h}} l_{\mathrm{h}} \leq 1$, where $L_{\mathrm{cx}}$ is the total path length across the CX layer. For a line of sight which intersects the interface region with an incidence angle $\theta$ and a spherical geometry (see Fig. 1), the total CXE emission path length is $L_{\mathrm{cx}}=2 l_{\mathrm{cx}} \cos (\theta)^{-1}$.

The ratio between the CXE emission integrated along the interface $L_{\mathrm{cx}}$ and the emission of the hot gas integrated over a distance $L$ ( $L$ in units of $l_{0}=1 \mathrm{pc}$ ) scales as:

$$
\begin{aligned}
P_{\mathrm{cx}} L_{\mathrm{cx}} / P_{\mathrm{h}} l_{0} L \approx & 1.2 \times 10^{-3} \\
& \times \cos (\theta)^{-1} \epsilon \alpha n_{\mathrm{c}} V_{\mathrm{r}} /\left(5.8 \times 10^{-14} n_{\mathrm{e}}^{2} \chi_{T, a} L l_{0}\right) \\
\approx & 1.2 \times 10^{-8} \epsilon \alpha \chi_{T, a}^{-1} \cos (\theta)^{-1}\left(n_{\mathrm{c}} V_{\mathrm{r}} n_{\mathrm{e}}^{-2}\right) L^{-1} .
\end{aligned}
$$

One can define a hot gas equivalent distance $L$ corresponding to equal contributions of charge-exchange and hot gas thermal emission:

$$
\begin{aligned}
& L(\mathrm{pc})=0.6 \times 10^{-8} \epsilon\left(\cos (\theta)^{-1} \alpha \chi_{T, a}^{-1}\right)\left(n_{\mathrm{c}} V_{\mathrm{r}} n_{\mathrm{e}}^{-2}\right) \text { or } \\
& L(\mathrm{pc})=0.06 \epsilon\left(\cos (\theta)^{-1} \alpha \chi_{T, a}^{-1}\right)\left(n_{\mathrm{c}} n_{\mathrm{e}}^{-2}\right)\left(V_{100}\right)
\end{aligned}
$$

where $V_{100}$ is in units of $100 \mathrm{~km} \mathrm{~s}^{-1}, n_{\mathrm{c}}$ and $n_{\mathrm{e}}$ in $\mathrm{cm}^{-3}$. This is equivalent to estimating an apparent or equivalent emission measure of the hot gas:

$$
E M_{\text {app }}=0.06 \epsilon\left(\cos (\theta)^{-1} \alpha \chi_{T, a}^{-1}\right) n_{\mathrm{c}} V_{100} \mathrm{~cm}^{-6} \mathrm{pc} \text {. }
$$

The contribution evidently increases with the relative flux of the neutrals, and increases as the hot gas density decreases, due to the emissivity function of the hot gas. A simple order of magnitude of the maximum CXE contribution for normal incidence and one crossing can be estimated by assuming that $\alpha$ and $\chi_{T, a}$ are of the order of unity, which gives

$L(\mathrm{pc})=0.03 \epsilon n_{\mathrm{c}} n_{\mathrm{e}}^{-2}\left(V_{100}\right)$

and

$E M_{\text {app }}=0.03 \epsilon n_{\mathrm{c}} V_{100} \mathrm{~cm}^{-6} \mathrm{pc}$.

Comparing this number with the hot gas emission measure allows us to infer whether it is worthwhile to calculate the $\mathrm{CX}$ emission in more detail.

\section{Type la supernova remnant in the Sedov phase}

We consider the case of a blast wave propagating in the ISM and generated by a Type Ia supernova in the Sedov phase. For this type of SNR the pre-shock ambient ISM may have not been fully ionized prior to the shock passage by the progenitor of the SNR. We assume a spherically symmetric blast wave, which propagates in a homogeneous dense, cool gas. We do not consider any detailed physics governing the density and temperature profile in the shock region, at variance with Wise \& Sarazin (1989), nor the structure of the shock, including the distribution of hot ions, which are not instantaneously created beyond the shock. This is beyond the scope of this paper. However we consider the effects of a simple spherical geometry shown in Fig. 1. Except when tangent to the interface region, whose thickness has been plotted out of scale (otherwise it is too narrow to be seen), an LOS with an impact parameter $p$ crosses it twice.

In the following example we use the physical characteristics of the supernova remnant DEM L71, a $\approx 4000$ yrs old and extremely regular Type Ia supernova remnant located in the LMC. It has been intensively observed with Chandra and recently analysed in great detail by Hughes et al. (2003), Rakowski et al. (2003) and Ghavamian et al. (2003). The outer blast wave of radius $r \approx 8$ pc propagating in the partially neutral ISM is traced by its X-ray emission, whose distribution is remarkably similar to the H-alpha pattern. Models fitted to both X-ray and optical data imply a shock velocity of about $v_{\mathrm{H}}=1000 \mathrm{~km} \mathrm{~s}^{-1}$, a shocked hot gas temperature $T_{\mathrm{h}}=4 \times 10^{6} \mathrm{~K}$ and the interaction with a partially neutral medium of density $n_{\mathrm{c}} \simeq 0.5 \mathrm{~cm}^{-3}$.

For the above value of the hot gas temperature and for a relative velocity of about $750 \mathrm{~km} \mathrm{~s}^{-1}$ between the shocked wind and the dense gas the filtration factor $\epsilon$ is close to 1, i.e. all neutrals in the pre-shock medium and crossing the fast shock are potential charge-exchange actors over a distance of the order of the CX mean free path, if we assume that they have survived a CR precursor and photoionization prior to the explosion. After the shock, the gas of initial density $\varepsilon n_{\mathrm{c}}$ starts to be heated by waves and collisions associated with the shock and the hot gas. The flux across the shock is of the order of $\varphi_{\mathrm{w}} \simeq n_{\mathrm{h}}$ $v_{\mathrm{r}} \approx 1.5 \times 10^{8} \mathrm{~cm}^{-2} \mathrm{~s}^{-1}$. We also assume that the combination of the high charge state ion distribution in the $0.5 \mathrm{keV}\left(4 \times 10^{6} \mathrm{~K}\right)$ gas and the reduced abundances (by a factor of $\approx 3$ with respect to solar) of the LMC type gas results in a global CXE crosssection about as high as in the $1-2 \times 10^{6} \mathrm{~K}$ solar abundance coronal plasma (i.e. $\alpha=1$ ). The CXE volume emissivity becomes:

$$
\begin{aligned}
P_{\mathrm{cx}} & \approx \Sigma_{0} \epsilon \alpha n_{\mathrm{c}} \varphi_{\mathrm{w}} \approx 6 \times 10^{-19} n_{\mathrm{c}} \varphi_{\mathrm{w}} \\
& =4.5 \epsilon \alpha 10^{-11} \mathrm{keV} \mathrm{cm}^{-3} \mathrm{~s}^{-1} .
\end{aligned}
$$

In contrast, a Raymond \& Smith type gas at $T=0.47 \mathrm{keV}$ (the fitted temperature to the Chandra spectra of the outer rim of DEM L71), with the LMC reduced abundances, and with $n_{\mathrm{e}}=2 \mathrm{~cm}^{-3}$, (i.e., the density behind the shock), has $P_{\mathrm{h}} \simeq 10^{-14} \mathrm{keV} \mathrm{cm}^{-3} \mathrm{~s}^{-1}\left(\chi_{T, a}=1 / 2.5\right)$ in the $0.1-0.5 \mathrm{keV}$ range. Applying Eq. (1) from the last section to $V_{100}=7.5$ $\left(V=750 \mathrm{~km} \mathrm{~s}^{-1}\right), n_{\mathrm{c}}=0.5 \mathrm{~cm}^{-3}, \alpha=1 n_{\mathrm{e}}=2 \mathrm{~cm}^{-3}$, $\chi_{T, a}^{-1}=2.5$ the equivalent path length is $L \approx 0.15 \mathrm{pc}$, or equivalently the emission measure is $\approx 0.5 \mathrm{~cm}^{-6} \mathrm{pc}$.

For an LOS impact parameter $p$ such that $p=0.95 r$ (see Fig. 1), the LOS through the hot gas is 5 pc. For emissions to balance, the extent of the CXE region along the LOS must be of the order of 30 times the normal incidence interface, while it is only 3.2 times this length. Here there is a large factor of $\approx 10$ missing for the two emissions to balance at $p=0.95 r$ in this simplistic approximation. The same calculation shows that the balance occurs for $p \approx 0.99$. This would mean, if $\varepsilon \alpha$ is of order 1, that the CXE emission becomes important only for linesof-sight that are exactly tangential to the interface. Figure 2 shows how the two integrated emissions vary as a function of the impact parameter $p$ in the soft X-ray range, assuming that for the hot gas the temperature decreases as $\frac{r}{r_{\mathrm{s}}} \frac{-4}{3}$, and the 


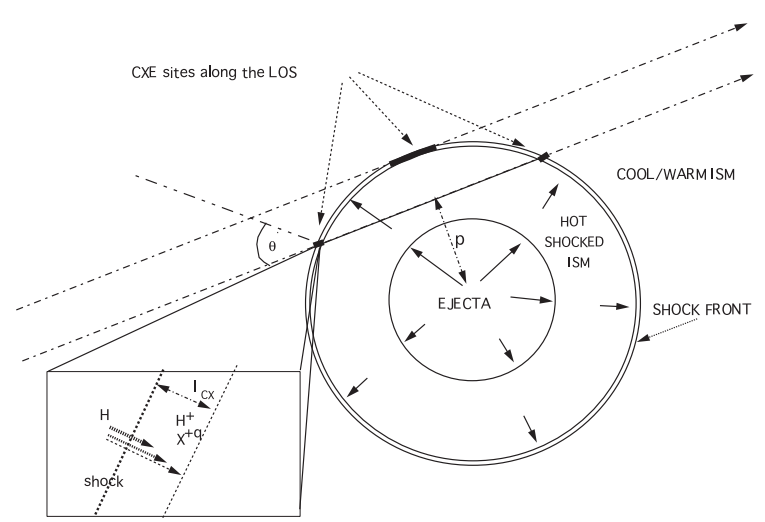

Fig. 2. Geometry used for the simplistic case of the spherical expansion of an SNR blast wave. The CXE interface region is not at scale.

density increases as $\frac{r}{r_{\mathrm{s}}}$, following the model results of Chevalier (1974).

The above estimate is an upper limit, which applies to light elements and the lower charge states, since we have implicitly assumed that the gas is immediately heated up at the post-shock temperature while this is not the case. Collisions increase the temperature gradually and high ion stages are deeper inside the remnant compared to lower state ions, also fully stripped heavy elements requiring a large number of ionizations appear far beyond the shock. i.e., where neutrals have disappeared. These effects are included in the calculations of Craig \& Sarazin (1989), and explain why, in their results, the relative contribution of the CX emission decreases strongly with the charge state and the elemental weight. In addition, while these authors have assumed electron-ion equilibration, this is not the actual case, as shown by detailed models (Raymond et al. 1995; Laming et al. 1996), which reinforces the previous effect, and reduces the charge-exchange contribution. Wise \& Sarazin (1989) find that the flux ratio reaches a maximum of $\approx 10^{-1}$ for helium-like emission of carbon and nitrogen, and typically averages $10^{-3}$ for emissions of $\mathrm{C}, \mathrm{N}$, and $\mathrm{O}$. Our simplistic calculation result is thus roughly equivalent in the case of helium-like emission of the lighter elements.

Geometrical effects can amplify the emission (see Fig. 2) simply because the soft X-ray emission from directions which are tangential to the interfaces is generated along a larger distance across the narrow layer. This could be tested with high resolution spectra taken at varying impact parameters along the edge of the shock front, using the fact that charge-exchange spectra have different characteristics than hot gas classical emission models. However, from the quantitative point of view, in this first test case the phenomenon is of negligible or minor importance.

\section{Galactic winds and halo cold clouds}

The powerful winds produced by starburst galaxies have been shown to interact with cool halo clouds, either preexisting clouds, or clouds carried into the halo by the wind itself (Suchkov et al. 1994; Lehnert et al. 1999; Strickland et al. 2002). The existence of numerous $\mathrm{H} \alpha$ filaments associated with $\mathrm{X}$-ray emitting gas, sometimes at a very large distance from the

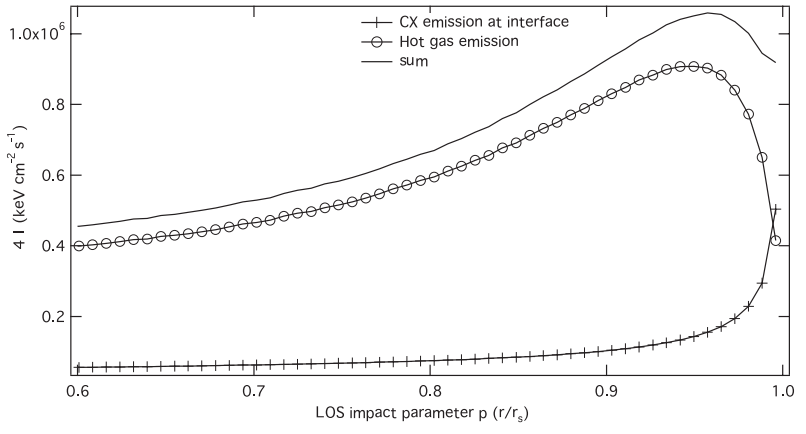

Fig. 3. Rough estimates of charge-exchange and thermal emission as a function of the impact parameter in the simple model of DEML71.

galaxy core, has motivated recent high spatial resolution observations of these regions, and combined optical and X-ray data have been used to develop models of the superwind/halo clouds interaction. According to the models favoured in the above studies, the expanding hot gas is driving a shock wave inside the engulfed cool cloud, and is itself decelerated and heated by a shock, which precedes the contact discontinuity with the cloud boundary. The shocked cloud is recombining and emitting $\mathrm{H}$-alpha, while the shocked superwind emits X-rays (e.g. Cecil et al. 2002; McDowell et al. 2003). Figure 1 (left) recalls the general structure of such interfaces, similar to the structures of the interstellar bubbles blown by stellar winds in cool or warm ISM (Weaver et al. 1977).

Here the source of the superions is the hot $10^{6} \mathrm{~K}$ gas formed beyond the shock that the expanding superwind must experience before interacting with the cool gas. The source of the neutrals is the shocked interstellar gas. If the cloud is initially cold and if the shock velocity jump is not too large the postshock gas can remain neutral. Alternately, the shock may be stronger and the shocked gas fully ionized, but radiative cooling in the dense swept-up shell may cause rapid recombination if the density is high enough (Weaver et al. 1977). One can expect in these conditions a charge-exchange interaction region at the expanding discontinuity between the shocked wind and the dense, cool gas. To be able to charge-exchange, neutrals must go through the heat conduction front which precedes the hot gas. This region is characterized by a strong temperature increase (and the subsequent progressive ionization) and a corresponding density decrease. As mentioned in Sect. 2, the number of neutrals $\epsilon n_{\mathrm{c}}$ succeeding in diffusing within this front and exchanging charges with hot ions must be large enough to produce a competitive X-ray emission source. At variance with the SNR blast wave case, $\epsilon$ is not of order unity but smaller. A realistic estimate of this filtration factor requires a detailed calculation of the ionization structure of this region,which must be described in a self-consistent manner for the plasma and the neutrals. This is beyond the scope of the paper, and we use the simple estimates of the filtration factor from Sect. 2, i.e. $\epsilon=\approx \frac{\sigma V_{\mathrm{r}}}{q_{i}}$.

We take as an example the case of the archetypal starburst galaxy NGC 253, which has been the subject of extensive Chandra observations (Strickland et al. 2002). The estimated "superwind" energy injection is $10^{42} \mathrm{erg} \mathrm{s}^{-1}$, which translates into about $10^{50}$ protons $\mathrm{s}^{-1}$ for an average initial wind 
velocity of $1000 \mathrm{~km} \mathrm{~s}^{-1}$. Assuming a very idealistic geometry, i.e. spherical expansion of a homogeneous wind, the average superwind flux at $2 \mathrm{kpc}$ from the center, the mean distance of the X-ray halo, can be of the order $2.5 \times 10^{5} \mathrm{~cm}^{-2} \mathrm{~s}^{-1}$.

For a flow initially at $1000 \mathrm{~km} \mathrm{~s}^{-1}$ and a surface discontinuity moving at $250 \mathrm{~km} \mathrm{~s}^{-1}, \varphi=2.5 \times 10^{5} \mathrm{~cm}^{-2} \mathrm{~s}^{-1}$ corresponds to $n_{\mathrm{e}}$ of the order of $10^{-2} \mathrm{~cm}^{-3}$. We assume pressure equilibrium at the conductive front which separates the hot shocked gas at $T \approx 10^{6} \mathrm{~K}$ from the dense shell, and the characteristics of the shell to be $T_{\mathrm{c}}=10^{4} \mathrm{~K}, n_{\mathrm{c}}=1 . \mathrm{cm}^{-3}$. The general formula of Sect. 2 gives for the equivalent hot gas path length:

$L=3 \times 10^{-2}\left(\epsilon \alpha \chi_{T, a}^{-1}\right)\left(n_{\mathrm{c}} V_{100} n_{\mathrm{e}}^{-2}\right)$

or

$$
\begin{aligned}
& E M_{\text {app }}=3 \times 10^{-2}\left(\epsilon \alpha \chi_{T, a}^{-1}\right)\left(n_{\mathrm{c}} V_{100}\right) \\
& \text { i.e. } L=\left(\epsilon \alpha \chi_{T, a}^{-1}\right) * 750 \mathrm{pc}=\alpha \chi_{T, a}^{-1} * 150 \mathrm{pc} \\
& E M_{\text {app }}=0.075\left(\epsilon \alpha \chi_{T, a}^{-1}\right) \mathrm{cm}^{-6} \mathrm{pc}=\left(\alpha \chi_{T, a}^{-1}\right) * 0.015 \mathrm{~cm}^{-6} \mathrm{pc} .
\end{aligned}
$$

This very large value suggests that in this case even a very small fraction of diffusing neutrals emits as much as a significant path length through the hot gas. As an example, for $\alpha \chi_{T, a}^{-1}$ of the order of unity and normal incidence, the CXE interface emission is equivalent to $150 \mathrm{pc}$ of hot gas if the fraction $\epsilon$ of diffusing neutrals is $\approx 2 \times 10^{-1}$, and the apparent emission measure is $0.015 \mathrm{~cm}^{-6} \mathrm{pc}$. Because actual structures are complicated, with cool clouds with sheetlike structures randomly distributed in the outer halo, and LOS may be at grazing incidence, the resultant emission may be of the same order as the total emission observed towards the halo, and may mimic diffuse emission distributed throughout the hot gas volume, with enhancements close to the denser clouds traced by Halpha.

Another prototypical starbust/superwind galaxy is M 82 . Recently a ridge of $\mathrm{H}$-alpha and $\mathrm{X}$-ray emission was discovered at a distance of $11 \mathrm{kpc}$ from the galaxy. Lehnert et al. (1999) have used the spectral X-ray properties and the two emission patterns to investigate the possible scenarios for this halo ridge of emission. The preferred scenario is the encounter of a massive ionized cloud with the galactic superwind. The wind is decelerated by a strong shock, and drives a relatively slow shock into the dense cloud. In their modelling the density and temperature in the shocked cloud are about $0.1 \mathrm{~cm}^{-3}$ and $T=10^{4} \mathrm{~K}$, the wind velocity is of the order of $800 \mathrm{~km} \mathrm{~s}^{-1}$, and the density in the wind is of the order of $10^{-3} \mathrm{~cm}^{-3}$. However, to be compatible with the HI radio data (Yun et al. 2003) the cloud has to be fully ionized, i.e. the neutral fraction $f_{\mathrm{n}}$ must be smaller than 0.035 . For this reason this case seems much more challenging. In the same way as above, the CXE contribution can be estimated to correspond to: $L=1200 * f_{\mathrm{n}}$ pc $\left(E M=0.24 * f_{\mathrm{n}} \mathrm{cm}^{-6} \mathrm{pc}\right)$, i.e. using the maximum neutral fraction, $L(\max ) \approx 45 \mathrm{pc}$. This is less than $10 \%$ of the thickness of the ridge $(0.9 \mathrm{kpc})$. However, there are several possibilities to reach an emission comparable to the observed emission attributed to the hot gas itself, i.e. for $L$ to reach the kpc order. First, the interface may be seen tangentially, in such a way that the signal is amplified by a factor of 10 . Second, there may be cloud sheets and several distinct interfaces. Third, and more probable, the requirements of the cloud properties in the proposed scenario are based on the assumption that the X-ray emission originates in shocked hot gas filling all the volume, and in contact with a single cloud. If there are a number of smaller clouds of density, say, $5 \mathrm{~cm}^{-3}$ instead of $0.1 \mathrm{~cm}^{-3}$, and if each cloud has an X-ray emitting interface, the sum of the interfaces can be at the origin of a large fraction of the observed emission, and there is no longer the need for large volumes of shocked and heated wind to explain the emission. In turn, this modifies the requirements for the cool/warm gas and its extent, allowing for denser and less ionized gas with a smaller filling factor, in agreement with the very high clumpiness implied by $\mathrm{H}$-alpha measurements.

The CX emission, if actually contributing in a nonnegligible manner, could explain the excellent correlations with $\mathrm{H} \alpha$, observed at any distance, including the very distant halo, and the small shifts between the $\mathrm{H}$-alpha shells and the X-ray enhancements. Also, because the CXE emission has a different spectrum, the abundances derived from fitting the low resolution spectra with a classical hot gas emission model will be different. Work is needed on the spectral characteristics of the CX emission to get better constraints and investigate whether or not the unphysically low iron abundances found in starburst halos (Strickland et al. 2002) can be linked to additional CX emission.

We have assumed in the above estimates of the maximum contribution that the ion stages are homogeneously distributed throughout the hot gas, including the interface. This is ideally the case in very sharp interfaces. In reality, there may be intermediate layers where recombination of hot ions occurs. In this case, the CX processes will be more frequent with hydrogenic ions penetrating deeper in the cold gas than fully stripped ions, resulting in fewer hydrogenic than helium-like lines. In the same way, light element emission lines can be favored. The combination of thermal emission and CXE emission can thus produce a resultant spectrum very different from classical or NEI models, which could explain the dominance of soft emission in the halo, and the correlation between softness and low iron abundance. Finally, because only starburst galaxies produce superwinds, this could also explain why low iron abundances found for halo gas are restricted to those types of galaxies, as noted by Strickland et al. (2002), and confirmed by the most recent Chandra observations.

\section{High velocity clouds in the galactic halo}

In our galactic halo, high velocity HI clouds (and some intermediate velocity clouds) have been found to be associated with enhanced soft X-ray emission (Hirth et al. 1985; Herbstmeier et al. 1995; Kerp et al. 1994, 1996, 1999), and also H-alpha emission (Tufte et al. 1998). The interaction between the fast cold gas of the HVC's and the hot gas from the halo is without any doubt at the origin of the emission, but the exact mechanism is still debated. The halo gas itself can play the same role as the shocked galactic wind in the above section. Instead of an outflowing galactic wind interacting with clouds at rest with respect to the galaxy, here rapidly falling clouds 
interact with a static halo hot gas. If neutrals diffuse through the conductive interface between the cold gas and the ambient halo hot gas, then CXE must be produced and may be at the origin of the observed soft X-ray excess. We use here numbers derived from Kerp et al. (1999) for complex C. Cloud velocities are of the order of $100 \mathrm{~km} \mathrm{~s}^{-1}$, and estimated cloud densities are $\approx 1 \mathrm{~cm}^{-3}$. This HVC complex has been shown to be closer than about $1 \mathrm{kpc}$, and therefore we assume the temperature and the density of the hot gas to be of the order of $T=10^{6} \mathrm{~K}$ and $n_{\mathrm{e}}=10^{-2} \mathrm{~cm}^{-3}$. For these numbers, a rough estimate of the CXE equivalent hot gas path length is:

$L=\approx 60$ pc if $\epsilon=2 \times 10^{-1}, \alpha=1$ and normal incidence,

and

$E M_{\text {app }}=6 \times 10^{-3} \mathrm{~cm}^{-6} \mathrm{pc}$.

Because the emission is likely to be enhanced due to the presence of clumps and because some lines of sight are at grazing incidences, the total $\mathrm{CX}$ emission could be a few times this value, i.e. reaching $E M=0.01 \mathrm{~cm}^{-6} \mathrm{pc}$. As a comparison, the average soft X-ray emission measure attributed by Kerp et al. (1999) to the complex C clouds is about $0.02 \mathrm{~cm}^{-6} \mathrm{pc}$. The similarity of the numbers suggests that CXE could play an important role in the HVC's soft X-ray emission. However, here the small size of the clouds is such that hot gas will flow around the engulfed and heated cloud, creating a head-tail structure. The diffusion of the neutrals through the contact discontinuity can be enhanced in this case, as in the heliospheric case, but such a complex structure will require sophisticated modelling.

\section{Galaxy clusters and cooling flows}

The observed X-ray emission from the central regions of a massive galaxy cluster is so intense that it implies cooling of the intracluster gas on timescales shorter than the cluster lifetime, and thus the formation of a cooling flow accumulating gas at the cluster center (Fabian \& Nulsen 1977; Cowie \& Binney 1977; Ferland et al. 1994). For a recent review see Fabian (1994). However, attempts to detect the cold condensed clouds have been unsuccessful, since atomic and molecular gas can only account for a small fraction of the deposited mass (Edge $\&$ Frayer 2003). Over the last few years, there has been increasing evidence from XMM and Chandra observations that this scenario needs some revision. The spectra of the cooling gas cannot be reproduced by cooling flow models: observations show that central, supposedly rapidly cooling cores do not emit $\mathrm{X}$-rays at temperatures below one third of the ambient temperature $T_{0}$ (e.g. Fabian et al. 2001, 2002; Peterson et al. 2003; Kaastra et al. 2003). The failure to detect intermediate temperature gas has led to a newly emergent view about the mass deposition which favors a much smaller deposition rate, now possibly compatible with the amount of cold gas detected e.g. under the form of CO (Edge \& Frayer 2003). In parallel, $21 \mathrm{~cm}$ studies show that fluctuations in the foreground are unlikely to be the cause of the soft X-ray and EUV excesses (Barnes \& Nulsen 2003).
These difficulties may point to the existence of a nonclassical X-ray emission process. Such a mechanism might possibly have some connection with the diffuse EUV excess detected in some clusters (Bowyer et al. 1999), whose origin is still actively debated (e.g., Berghofer \& Bowyer 2002). Because CXE emission in some respects mimics hot gas cooling, it may be worthwhile to investigate whether hot/cool gas interfaces can contribute a fraction of the observed emission. In this respect we note that: (i) There is a number of observations that show a tight spatial correlation between optical filaments and soft X-ray emission, e.g. the recent detailed maps obtained with Chandra of the Perseus cluster (Fabian et al. 2003), or 2A0335+096 (Monroe \& Gelderman 2003). (ii) There is probably a link between these correlations and the trends found in the past by Heckman et al. (1989) and more recently by Peres et al. (1998) which relate $\mathrm{H} \alpha$ luminosities and inferred mass deposition (i.e. X-ray emission). (iii) Using optical line ratios and linewidths, Crawford \& Fabian (1992) have demonstrated that the most plausible origin for the optical emission in cooling flow nebulae is a large number of warm gas interfaces formed in mixing layers. These mixing layers surround cold clouds embedded in the hot X-ray emitting gas which fills the cores of the cooling flows. In parallel, from studies of the physical conditions in the cooling flow centers, Ferland et al. (2002) suggest that large masses of cold dense gas, in addition to the warmer molecular gas detected recently, could indeed be present. If those mixing layers are indeed so numerous, and associated with the X-ray emission, as suggested by the combination of (i)-(iii), then charge-exchange between cold gas and relatively moving hot gas could occur in the interfaces and generate X-rays.

Rough estimates of the CXE emission at interfaces between hot intracluster medium (ICM) and cool clouds at cluster centers can be obtained as in the previous section, assuming cold gas moves relative to hot gas at velocities of the order of $100 \mathrm{~km} \mathrm{~s}^{-1}$, as inferred from the optical linewidths (e.g. Peres et al. 1998). One again expects the cold gas to be shocked and heated by the hot gas, and the existence of a conduction front at the gas discontinuity, which neutral atoms must cross to produce the charge-exchange emission.

Plasma densities of the hot emitting gas are of the order of $10^{-3 ;-4} \mathrm{~cm}^{-3}$ in most clusters (Fabian 1994) and temperatures are of the order of $10^{7} \mathrm{~K}$. Cool gas densities may be as high as $10^{1} \mathrm{~cm}^{-3}$ (Crawford \& Fabian 1992). Those numbers correspond to optimal conditions of CXE production according to Sect. 2. Equation (1) shows that for $n_{\mathrm{c}}=0.1 \mathrm{~cm}^{-3}$ and $n_{\mathrm{e}}=10^{-4} \mathrm{~cm}^{-3}$ to ensure pressure equilibrium between warm $(10000 \mathrm{~K})$ and hot $\left(10^{7} \mathrm{~K}\right)$ gases, and for $V_{\mathrm{r}}=100 \mathrm{~km} \mathrm{~s}^{-1}$, the hot gas equivalent distance is

$L(\mathrm{pc})=3 \times 10^{-2} \epsilon\left(\alpha \chi_{T, a}^{-1}\right)\left(n_{\mathrm{c}} n_{\mathrm{e}}^{-2}\right)\left(V_{100}\right)$

$L=\left(\epsilon \alpha \chi_{T, a}^{-1}\right) * 3 \times 10^{5} \mathrm{pc},=\left(\alpha \chi_{T, a}^{-1}\right) * 0.6 \times 10^{5} \mathrm{pc}$ if $\epsilon \approx 0.2$

and

$E M_{\text {app }}=3 \times 10^{-2} \epsilon n_{\mathrm{c}} V_{100} \approx 0.5 \times 10^{-3} \mathrm{~cm}^{-6} \mathrm{pc}$.

This estimate of the maximal contribution evidently leads to very high numbers. A fraction of diffusing neutrals much 
smaller than what we have used seems to be large enough to create a non negligible emission. Even slower clouds could also play a role. If there are numerous cold clouds moving in the intra-cluster hot gas, as inferred by Crawford \& Fabian (1992) to reproduce the optical emission, their associated chargeexchange emission could mimic diffuse emission from the hot gas itself. This shows that more realistics models are definitely needed.

If, as suggested by the above estimates, there is CXE emission in the cold-hot gas interfaces and if this source is a non negligible contributor to the total X-ray emission, then the interpretation of the diffuse X-ray emission in terms of global cooling leads to an overestimate of the cooling strength, i.e. of the deposited mass. As a matter of fact there is no global cooling of the entire volume of hot gas linked to this emission. The fraction of the emission due to charge-exchange cools the hot gas at some specific locations, namely the interfaces with cool or warm gas, and is an accelerated conversion to X-ray photons of the potential energy stored in the ions, where cold clouds and the hot gas have the relative motions which inject the neutrals into the ions. The CXE brightness distribution is thus a picture of the instantaneous dynamics, it cannot be extrapolated back in time as global cooling constant with time.

\section{Conclusions}

The X-ray emission associated with the charge-exchange process between high ions and neutrals at interfaces between hot moving plasmas and cool ISM is parameterized and estimated on the basis of simplistic models, and its magnitude is compared with hot gas thermal emission. Because the relative importance of the CXE brightness is proportional to the quantity $n_{\mathrm{n}} U n_{\mathrm{e}}^{-2}$, where $U$ is the relative velocity of the hot gas with respect to the partially neutral gas, $n_{\mathrm{n}}$ the neutral density, and $n_{\mathrm{e}}$ the hot gas density at the interface, the CXE X-ray emission will be increasingly important in the most rarefied plasmas, and where the highest density contrasts occur.

In conduction fronts, the fraction of neutrals diffusing within the hot gas and the superions before being ionized is a factor $\epsilon$, estimated in a very crude manner to be of the order of $10^{-1}$. Although the CXE emission is generated in very small envelopes, whose thickness corresponds to the mean free path of the neutrals within the hot gas, we find that it may be non negligible, even for values of $\epsilon$ as small as $10^{-1}$ in the cases of galactic superwinds interacting with an ambient neutral gas, of high velocity clouds in the halo, and of intra-cluster hot gas interacting with cold or warm clouds, at least in the frame of these simple models. The emission from the envelopes of Type Ia supernova remnants is negligible, although CXE could slightly increase the limb brightening effects in some particular sightlines.

High velocity $\left(V \leq 100 \mathrm{~km} \mathrm{~s}^{-1}\right.$ ) clouds in the galactic halo can be used as a test case for the CX emission, because they are observed with great detail and flow into hot gas of well known characteristics. Our simple and very crude estimate of the emission, i.e. an equivalent $E M$ of $0.006 \mathrm{~cm}^{-6} \mathrm{pc}$ in the soft $\mathrm{X}$-ray range per interface at normal incidence, is very similar to the observed $E M$ of about $0.02 \mathrm{~cm}^{-6} \mathrm{pc}$ for a number of Complex C clouds (Kerp et al. 1996, 1999).

These results call for detailed models establishing selfconsistently plasma and neutrals density and velocity distributions across the hot-cool gas interfaces. The difficulty with these models is the need for kinetic descriptions of the neutrals, because the mfp of neutrals can be of the order of the size of the interface. According to the models developed for the heliosphere, the inclusion of the neutrals in a self-consistent manner modifies the interfaces significantly (Baranov \& Malama 1991).

Because the spectra associated with the CXE emission are different from those of cooling hot gas, namely they have no continuum, and different line ratios, further investigations on how CXE emission may influence the spectral properties of $\mathrm{X}$-ray astrophysical plasmas and their interpretations appear to be an interesting task. In particular, if spectra are contaminated by CXE emission, their interpretation in terms of hot gas classical radiation may be partially misleading. On the luminosity aspect, because the LOS intensity does not scale with the emission measure, but instead depends on the characteristics of the interfaces and their numbers, $\mathrm{CX}$ emission may also alter the interpretation in terms of cooling and hot gas density. In future, hopefully highly resolved X-ray spectra (McCammon et al. 2002) will distinguish between the different types of X-ray emission.

Acknowledgements. I am indebted to J. Raymond for a large number of suggestions and a critical analysis which led to considerable improvement of this paper. Many thanks to him.

I also thank the anonymous referee for useful remarks and corrections.

\section{References}

Baranov, V. B., \& Malama, Y. G. 1993, J. Geophys. Res., 98, 15157

Barnes, D. G., \& Nulsen, P. E. J. 2003, MNRAS, 343, 315

Berghöfer, T. W., \& Bowyer, S. 2002, ApJ, 565, L17

Bowyer, S., Berghöfer, T. W., \& Korpela, E. J. 1999, ApJ, 526, 592

Cecil, G., Bland-Hawthorn, J., \& Veilleux, S. 2002, ApJ, 576, 745

Chevalier, R. A. 1974, ApJ, 188, 501

Cox, D. 1998, The Local Bubble and beyond, ed. Breitschwerdt et al. Lect. Notes Phys., 506 (Berlin: Springer Verlag), 121

Cowie, L. L., \& Binney, J. 1977, ApJ, 215, 723

Cravens, T. E. 1997, GRL, 24, 105

Cravens, T. E. 2000, ApJ, 532, L153

Cravens, T. E. 2002, Science, 296, 1042

Cravens, T. E., Robertson, I. P., \& Snowden, S. L. 2001, JGR, 106, 24883

Crawford, C. S., \& Fabian, A. C. 1992, MNRAS, 259, 265

Edge, A. C., \& Frayer, D. T. 2003, ApJ, 594, L13

Fabian, A. C., \& Nulsen, P. E. J. 1977, MNRAS, 180, 479

Fabian, A. C., Mushotzky, R. F., Nulsen, P. E. J., \& Peterson, J. R. 2001, MNRAS, 321, L20

Fabian, A. C., Allen, S. W., Crawford, C. S., et al. 2002, MNRAS, 332, L50

Fabian, A. C., Sanders, J. S., Crawford, C. S., et al. 2003, MNRAS, 344, L48

Ferland, G. J., Fabian, A. C., \& Johnstone, R. M. 2002, MNRAS, 333, 876 
Freyberg, M. J. 1994, Ph.D. Thesis

Ghavamian, P., Rakowski, C. E., Hughes, J. P., \& Williams, T. B. 2003, ApJ, 590, 833

Heckman, T. M., Baum, S. A., van Breugel, W. J. M., \& McCarthy, P. 1989, ApJ, 338, 48

Herbstmeier, U., Mebold, U., Snowden, S. L., et al. 1995, A\&A, 298, 606

Hirth, W., Mebold, U., \& Mueller, P. 1985, A\&A, 153, 249

Hughes, J. P., Ghavamian, P., Rakowski, C. E., \& Slane, P. O. 2003 , ApJ, 582, L95

Izmodenov, V. V., Geiss, J., Lallement, R., et al. 1999, J. Geophys. Res., 104, 4731

Jenkins, E. B. 2002, ApJ, 580, 938

Kaastra, J. S., Lieu, R., Tamura, T., Paerels, F. B. S., \& den Herder, J. W. 2003, A\&A, 397, 445

Kerp, J., Lesch, H., \& Mack, K.-H. 1994, A\&A, 286, L13

Kerp, J., Mack, K.-H., Egger, R., et al. 1996, A\&A, 312, 67

Kerp, J., Burton, W. B., Egger, R., et al. 1999, A\&A, 342, 213

Kharchenko, V., \& Dalgarno, A. 2000, JGR, 105, 18351

Lallement, R. 1998, in The Local Bubble and beyond, ed. Breitschwerdt et al., Lect. Notes Phys., 506 (Berlin: Springer Verlag), 19

Lallement, R. 2003, A\&A, in press

Lehnert, M. D., Heckman, T. M., \& Weaver, K. A. 1999, ApJ, 523, 575

Lisse, C. M., Dennerl, K., Englhauser, J., et al. 1996, Science, 274, 205

McCammon, D., Almy, R., Apodaca, E., et al. 2002, ApJ, 576, 188
McDowell, J. C., Clements, D. L., Lamb, S. A., et al. 2003, ApJ, 591, 154

Monroe, T. R., \& Gelderman, R. 2003, Am. Astron. Soc. Meet., 202

Peres, C. B., Fabian, A. C., Edge, A. C., et al. 1998, MNRAS, 298, 416

Peterson, J. R., Kahn, S. M., Paerels, F. B. S., et al. 2003, ApJ, 590, 207

Rakowski, C. E., Ghavamian, P., \& Hughes, J. P. 2003, ApJ, 590, 846

Raymond, J. C., \& Smith, W. 1977, ApJS, 35, 419

Robertson, I., Cravens, T., Snowden, S., \& Linde, T. 2001, Sp. Sci. Rev., 97, 401

Schwadron, N., \& Cravens, T. E. 2000, ApJ, 544, 558

Snowden, S., McCammon, D., Burrows, D. N., \& Mendenhall, J. A. 1994, ApJ, 424, 714

Snowden, S., Freyberg, M. J., Plucinsky, P. P., et al. 1995, ApJ, 454, 643

Snowden, S., Egger, R., Finkbeiner, D. P., Freyberg, M. J., \& Plucinsky, P. P. 1998, ApJ, 493, 715

Snowden, S., Freyberg, M., Kuntz, K., \& Sanders, W. 2000, ApJS, 128,171

Strickland, D. K., Heckman, T. M., Weaver, K. A., Hoopes, C. G., \& Dahlem, M. 2002, ApJ, 568, 689

Suchkov, A. A., Balsara, D. S., Heckman, T. M., \& Leitherner, C. 1994, ApJ, 430, 511

Tufte, S. L., Reynolds, R. J., \& Haffner, L. M. 1998, ApJ, 504, 773

Weaver, R., McCray, R., Castor, J., Shapiro, P. R., \& Moore, R. 1977, ApJ, 218, 377

Wise, M. W., \& Sarazin, C. L. 1989, ApJ, 345, 384 